\title{
L'évacuation des eaux usées de laiterie
}

\author{
par \\ A. ECK \\ Directeur de l'Association pour l'Etude de l'Utilisation \\ de l'Eau et l'Epuration des eaux usées \\ dans l'Industrie Laitière
}

Durant de longues années il était habituel à tout auteur traitant de l'évacuation des effluents industriels, et surtout s'il appartenait au milieu professionnel, de faire ressortir la contradiction existant entre les exigences des services chargés de la protection des milieux aquatiques, et celles qui avaient pour objet l'homme lui-même. Il apparaissait que le "poisson " était mieux défendu que l'homme.

Depuis quelques années le problème n'est plus de sauvegarder le milieu, mais simplement d'assurer à tous les êtres vivants une ressource suffisante en eau.

Si l'expression en est simple, les données en sont nombreuses et mettent en jeu des relations complexes. Le sujet en cause pourrait d'ailleurs servir d'exemple pour un exercice de "gouvernement ", s'il en existait comme il existe des « jeux d'entreprise ».

Maintenir la ressource, l'améliorer qualitativement et quantitativement, voilà les objectifs de la loi du 16 décembre 1964 relative au régime et à la répartition des eaux et à la lutte contre la pollution.

Il convient cependant de noter comme le fait une circulaire interministérielle du 23 mai 1968, que la loi du 16 décembre 1964 n’a pas abrogé les textes existants contenus en particulier dans le Code Rural, pas plus que la législation relative aux établissements classés... Nous y reviendrons.

Retenons pour l'instant que l'épuration demeure plus que jamais nécessaire.

\section{LES TRAITEMENTS D'EPURATION}

Ce serait faire injure à nos lecteurs que de leur rappeler les caractéristiques des eaux usées de laiterie. Au surplus, il a été nettement confirmé que chaque établissement représente un cas d'es- 
pèce, qu'il s'agisse de la composition, des débits, total, instantané, et de la charge polluante.

Nous verrons que cependant les Pouvoirs Publics ont établi des "forfaits de pollution ».

Dans la gamme des procédés visant à diminuer la pollution des liquides rejetés dans le milieu extérieur (ou dans un réseau public d'assainissement) par une usine laitière, il est probable que tous ceux que l'esprit peut concevoir ont été appliqués.

Toutefois une sorte de classement s'est opéré peu à peu, reposant sur des critères autant techniques qu'économiques, si l'on veut bien inclure dans l'économie. la facilité de contrôle et d'utilisation.

\section{L'épandage par aspersion}

Pendant longtemps l'épandage par aspersion est apparu comme la solution.

Certes, cette méthode rencontre des impératifs : surface nécessaire, capacité d'absorption des sols, équilibre N-P-K à corriger. Cependant le rendement peut atteindre $100 \mathrm{p} .100$, avec un minimum de main-d'œuvre.

Néanmoins la concentration industrielle fait apparaître les limites du procédé : en considérant qu'on peut épandre en moyenne $10 \mathrm{~m}^{3}$ d'eau sur 1 hectare, un établissement traitant 500000 litres / jour devrait disposer de 150 hectares pouvant être arrosés.

Cette surface peut être trouvée mais pas toujours. En outre d'autres contraintes apparaissent, liées aux distances. Le réseau de canalisations, la pression de refoulement, d'autant plus importants que les distances et les surfaces augmentent, correspondent à des investissements qui probablement, pour tel cas particulier, dépassent ceux entraïnés par le traitement proprement dit.

\section{Les traitements biologiques}

L'expérience montre que, seuls, les traitements biologiques sont susceptibles d'apporter une solution. Mais ces traitements doivent être d'une exploitation et d'un entretien faciles et être capables d'absorber les variations de charge et les à-coups de débit. En fait on s'adresse aux procédés désormais classiques, d'oxydation : boues activées, ou chenal d'oxydation, et qui tendent du reste à devenir à oxydation totale.

Toutes ces techniques impliquent :

- un brassage et une aération,

- une évacuation logique de l'effluent et éventuellement des boues en excédent. 
Le brassage et l'aération sont obtenus soit par des brosses de surface, soit des turbines, soit par un refoulement d'air à travers la masse d'eau à traiter.

La comparaison entre les trois systèmes est difficile, car si chacun a ses avantages et ses inconvénients, ceux-ci sont de poids différents selon les caractéristiques des eaux à traiter. En outre le chenal à oxydation totale peut, par exemple, éviter la construction d'un décanteur secondaire (final) : le coût annuel tiendra compte d'une dépense plus élevée d'énergie électrique (pour la rotation des brosses) mais d'un taux d'amortissement moins grand.

En bref, la comparaison ne peut être effectuée que sur un cas concret et encore doit-elle tenir compte des disponibilités financières de l'entreprise (trésorerie, possibilité de financement à court ou moyen terme...).

Le deuxième aspect évoqué à propos de l'oxydation, celui de l'évacuation de l'effluent et des boues revêt une grande importance, non pas tant au plan technique qu'à propos de la facilité de contrôle et de l'automatisation possible. Il serait même souhaitable que l'on puisse programmer la succession des opérations dès lors que le fonctionnement n'est pas continu.

Ceci n'est pas une vue de l'esprit : la régulation des installations de traitement a déjà reçu plusieurs applications ; les deux réglages fondamentaux du débit d'air (dans le système des boues activées) et des extractions de boue en excès sont confiés à un dispositif qui va du simple régulateur à l'ordinateur (calculateur analogique).

\section{Le problème des boues}

Sauf dans le cas d'oxydation totale, le traitement laisse des boues. Leur volume est évidemment fonction de la composition des eaux à traiter.

L'utilisation de ces boues se heurte à de nombreuses difficultés, dues en général à leur teneur en eau et à leur instabilité.

Le séchage à l'air (après un préséchage en "silo", ou non) a tendance à disparaître car il requiert surface et main-d'œuvre. Aussi, le séchage accéléré est parfois pratiqué (par filtration sous vide, par centrifugation, par filtration sous pression).

La digestion aérobie paraît devoir l'emporter sur la digestion anaérobie.

Mais l'incinération, d'autant plus intéressante que l'installation est plus grande, permet de résoudre totalement le problème. Une installation de ce type va fonctionner prochainement, sur des eaux de ville, il est vrai. Bien sûr, dans ce procédé il faut fournir de l'énergie pour sécher partiellement les boues et les brûler ensuite. Le bilan paraît cependant positif dès que l'on tient compte des 
contraintes diminuées, voire supprimées, d'encombrement et de main-d'œuvre.

Cet aspect se retrouve donc ici comme ailleurs chaque fois qu'il s'agit d'opérations industrielles.

\section{LEGISLATION ET REGLEMENTATION}

La lutte contre la pollution des eaux a pris récemment une importance considérable : il ne s'agit plus seulement répétons-le, de protéger le milieu dans lequel vit l'homme, ou le milieu aquatique, mais aussi et surtout de faire en sorte que l'homme ne manque pas d'eau, de cette eau dont il fait une consommation de plus en plus grande.

\section{La Loi du 16 décembre 1964}

Cette constatation pourrait amener à considérer que la loi du 16 décembre 1964 constitue la synthèse de dispositions législatives et réglementaires visant à réduire et réprimer la pollution.

Mais la circulaire interministérielle du 23 mai 1968 rappelle opportunément - on verra plus loin pourquoi - que « la loi de 1964 n'a pas abrogé les textes existants contenus notamment dans le Code Rural (1), le Code de la Santé Publique... la législation relative aux établissements classés... ».

De même qu'il ne suffit pas à un responsable d'établissement (ou d'entreprise) de se conformer aux dispositions découlant de la loi de 1917 pour échapper à l'application de l'article 434.1 du Code Rural, de même se soumettre aux obligations édictées, en vertu de la loi de 1964 par les Agences financières de bassin, ne signifie pas être à l'abri d'une répression éventuelle, fondée sur les autres textes.

Cette observation, pour évidente qu'elle paraît, doit cependant être rappelée, car trop souvent on constate une confusion née probablement de la désignation de la taxe appelée redevance-pollution qu'on assimile à un paiement ouvrant droit, en quelque sorte, à déverser des effluents polluants.

L'apport de la loi de 1964 consiste essentiellement dans le domaine qui nous intéresse ici, en la création d'organismes au niveau des six grands bassins français, chargés de lutter contre la pollution (et non la réprimer) c'est-à-dire de la diminuer en mettant en œuvre (ou faisant mettre en œuvre) les moyens nécessaires, apportés par un financement calculé sur la pollution constatée.

(1) Entre autres, l'article 434.1. 
Cependant, il faut noter que la loi de 1964 comporte également des dispositions permettant de rendre la répression plus sévère et d'autre part introduisant une innovation juridique considérable, par l'institution de " réparations civiles ".

Il faut aussi noter que deux phases successives sont prévues pour l'application des sanctions :

- condamnation à une peine contraventionnelle pour infraction et injonction au contrevenant d'avoir à exécuter les travaux ou aménagements utiles,

- en cas de non-exécution des travaux dans le délai prescrit, application de peines délictuelles, amende, assortie d'astreinte, voire d'emprisonnement ; le Tribunal pouvant « autoriser le Préfet à exécuter d'office les travaux nécessaires, le coût de ces travaux incombant à la personne physique ou morale dont le condamné est le préposé ou le représentant».

Cependant, des décrets d'application sont prévus par la loi : certains n'étant pas encore entrés en vigueur, aucune jurisprudence n'existe encore.

Puisque nous en sommes au domaine jurisprudentiel, nous pouvons signaler qu'aucun élément nouveau saillant n'est apparu depuis l'étude de M. Kempf (2).

\section{LES ORGANISATIONS DE BASSIN AGENCES FINANCIERES ET COMITES DE BASSIN}

La France ayant été divisée en six «bassins " (Artois-Picardie, Rhin-Meuse, Rhône-Méditerranée-Corse, Adour-Garonne, Loire-Bretagne et Seine-Normandie), à chacun de ces bassins, correspondent une Agence financière et un Comité de bassin.

Selon les termes du Sénateur, M. Lalloy, rapporteur sur le projet de loi, les Agences financières, établissements publics, " sont organi" quement chargés de faciliter les diverses actions d'intérêt commun " au bassin ou groupe de bassins. Leurs moyens d'action sont essen" tiellement d'ordre financier, et c'est par le jeu conjugué des rede"vances versées par les usagers de l'eau sur les prélèvements et sur " les déversements et des subventions ou prêts attribués aux per" sonnes de droit public et de droit privé qu'elles auront une action " déterminante à la fois d'incitation, de coordination et de contrôle

(2) Les Aspects juridiques de la pollution des cours d'eau par les entreprises industrielles. Editions de l'Eau.

On pourra également consulter avec profit l'exposé de $M$. Gautron (la lutte contre la pollution des eaux - Etat du droit actuel) aux Journées d'Etude de I'A.F.E.E. (novembre 1968). 
» sur les actions entreprises par les collectivités publiques de tous » ordres et sur les particuliers.

"Les Agences de bassin jouiront de l'autonomie financière. Elles " constitueront, à l'échelle du bassin, un rouage essentiel de la lutte " contre la pollution ".

Le Comité de bassin, lui, est consulté "sur l'opportunité des " travaux et aménagements d'intérêt commun envisagés dans la zone " de sa compétence, sur les différends pouvant survenir entre les " collectivités ou groupements intéressés et, plus généralement, sur "toutes les questions faisant l'objet de la présente loi ".

En fait la doctrine de ces organismes s'est peu à peu précisée au cours de l'année 1968, pendant laquelle se sont mis en place Agences et Comités.

\section{Les redevances}

Les moyens financiers des Agences proviennent en partie de dotations de l'Etat et essentiellement des redevances perçues auprès des « redevables".

Ces redevances sont assises d'une part sur la consommation en eau, et d'autre part sur la pollution rejetée dans le milieu extérieur.

On peut donc dire que sont taxés les prélèvements sur la " ressource " en eau qui l'altèrent quantitativement, et les rejets qui l'altèrent qualitativement.

Ainsi, les Pouvoirs Publics espèrent voir les prélèvements, sinon diminuer, du moins ne pas augmenter, et la pollution cette fois diminuer.

D'un autre côté, grâce à ces ressources, l'Agence peut provoquer, en les finançant en partie, les travaux et aménagements nécessaires à cet objectif primordial.

Concrètement, les choses sont plus compliquées, En effet, chaque bassin ne présente pas le même degré de pollution, ne dispose pas des mêmes ressources en eau, et surtout la pollution des bassins n'est pas due nécessairement aux mêmes causes.

Ainsi, chaque Comité ayant adopté un programme tenant compte des caractéristiques du bassin, l'Agence doit en répartir la charge financière entre les redevables, aussi bien au titre des "prélèvements » que de la "pollution ».

Enfin, les six bassins sont loin d'être homogènes, et dans chacun, des zones ont été découpées, correspondant à des caractéristiques diverses (répartition de la pluviométrie, régime des cours d'eau, nappes souterraines, industrialisation...).

Mais quand on rapporte ceci à l'industrie laitière, il y a plus grave. 
En effet, lorsqu'il s'est agit de répartir le montant d'un programme d'assainissement, c'est-à-dire de calculer pour chaque redevable le montant de la redevance pollution, il a fallu trouver d'abord une clé de répartition équitable, c'est-à-dire exprimant la part dans la pollution, des villes, des industries, de l'industrie chimique, de l'industrie sidérurgique, de la sucrerie, de la papeterie, de la laiterie...

L'équivalent-habitant ? Sa valeur est connue, qui ne permet pas d'établir de corrélations réelles entre les diverses activités industrielles.

Alors on a inventé une notion de "matières oxydables " (MOX), à laquelle on associe la quantité de matières en suspension (MES) et pour deux bassins, la pollution saline.

\section{Les « critères " de la pollution}

La quantité de MOX est déclarée égale au tiers de la somme de la DCO (demande chimique d'oxygène) et de deux fois la DBO5 (demande biochimique d'oxygène en cinq jours) :

$$
\operatorname{MOX}=\frac{\mathrm{DCO}+2 \times \mathrm{DBO} 5}{3}
$$

On voit immédiatement que pour les industries dont les eaux n'ont qu'une faible DBO5, la pollution " chimique " (pas nécessairement moins grave que la «biochimique ») est divisée par trois. Mais la formule n'est, paraît-il, pas définitive.

En outre, il est bien prévu qu'est taxée la «pollution ajoutée », c'est-à-dire celle égale à la différence entre la pollution constatée en aval et celle constatée en amont, et que chaque redevable peut faire mesurer cette pollution, à ses frais, il est vrai.

Pratiquement, compte tenu du nombre très grand d'établissements et surtout du nombre, restreint, de laboratoires susceptibles de procéder à ces mesures, on applique des forfaits.

Ainsi pour tout mètre cube de lait traité en un jour moyen du mois d'activité maximale, une laiterie, une beurrerie ou une fromagerie, ne rejetant ni sérum, ni babeurre, sont réputées apporter $3,4 \mathrm{~kg}$ de MOX et 1,2 kg de MES au milieu extérieur.

Pour chaque mètre cube de sérum ou de babeurre rejeté sans traitement, on ajoute $46 \mathrm{~kg}$ de MOX et $28 \mathrm{~kg}$ de MES.

On aboutit ainsi à des situations parfois aberrantes : l'usine centrale alimentée par plusieurs centres secondaires sera taxée deux fois ; le forfait ne tenant aucun compte de l'état des installations de traitement du lait (qui commande cependant le degré de pollution des effluents) les investissements visant à diminuer ce degré deviennent inutiles; enfin, l'Agence peut imposer la mesure de la pollution réelle aux redevables que le forfait favoriserait. 
Certes, la lutte contre la pollution est nécessaire : pour autant, il demeure non moins nécessaire de moduler le forfait, puisque forfait il y a, selon d'autres critères que le volume de lait traité.

Par exemple, nous savons bien que la pollution rejetée dépend, entre autres facteurs, des soins apportés au travail à l'intérieur de l'établissement même. Le forfait peut donc inciter à négliger ces soins qui coûtent souvent cher.

En revanche, il faut reconnaître deux éléments positifs : ce que nous avons dit du forfait (en MOX et MES) s'applique aux effluents non traités ; tout traitement même partiel entraîne un abattement de la redevance, qui peut aller dans certains cas jusqu'à 100 p. 100 (épandage bien fait par exemple, sauf dans l'Artois-Picardie !).

D'autre part, les ressources financières ainsi rassemblées sont destinées à servir au financement des installations, de traitement, projetées par les municipalités et les industriels.

Comme des exemples peuvent déjà être invoqués, ceci constitue une possibilité réelle.

\section{L'écrêtement}

D'un autre côté, l'Etat désireux de "soulager ", au moins la première année, les entreprises, a décidé de verser aux Agences une somme égale à 10 p. 100 des sommes qu'elles auront reçues des redevables, Cette subvention doit servir à " écrêter " les redevances individuelles.

A ce propos, on a dit que de la sorte, les contributions des entreprises seraient limitées à 1 p. 100 de la «valeur ajoutée ». En réalité il s'agit d'un taux moyen national résultant de calculs parfaitement arbitraires, et selon des données telles que la part de valeur ajoutée dans le produit national brut, le nombre d'établissements et la pollution théorique qu'ils entraînent.

En fait, chaque cas doit être examiné par l'Agence financière, qui doit tenir compte des situations particulières, en évitant de créer des situations intolérables.

De toute manière, il ne s'agit que de remboursement a posteriori, que peut demander l'entreprise qui se juge en péril.

Remarquons que cette notion de valeur ajoutée n'a pas de définition légale, sinon dans les textes d'application de l'ordonnance relative à la participation des travailleurs aux fruits de l'expansion, et que, complexité supplémentaire, elle doit, dans le domaine qui nous intéresse ici, être appréciée par établissement et non par entreprise.

On le voit, toutes les questions sont loin d'être résolues. Ainsi en est-il de la taxe dite d'assainissement. 


\section{La taxe d'assainissement}

Les Communes disposant d'un réseau d'assainissement sont autorisées à prélever une taxe correspondant au service rendu (transport et éventuellement traitement des effluents). Mais cette taxe a pour assiette la consommation d'eau. Or il n'y a qu'un lien très lâche entre consommation et pollution. En outre, la taxe est affectée de coefficients de minoration selon le volume d'eau consommé. Les entreprises peuvent donc être lésées et les municipalités aussi.

En tout cas, lorsqu'un établissement est alimenté en eau par le réseau municipal, et que les effluents dont il est la source sont déversés dans un réseau public, cet établissement n'est pas connu de l'Agence financière, et ne peut pas l'être : les questions relatives aux redevances (prélèvement et pollution) ne se traitent qu'entre la municipalité et l'Agence, quitte pour la première à calculer pour l'établissement en cause, la pollution dont il est responsable, et la redevance correspondante,

Quoi qu'il en soit, la taxe d'assainissement justifie une révision de son assiette.

Un autre aspect doit être souligné : les redevances que doit acquitter un établissement peuvent varier de 1 à 50 selon son implantation géographique.

Cette distorsion dans les charges financières peut faire naître un sentiment d'injustice et pourquoi pas ? le désir de modifier l'emplacement de certains établissements. Il ne peut en être question pour l'industrie laitière. Cependant, il y a là un aspect qui ne peut laisser indifférents les responsables de l'aménagement du territoire et de l'emploi.

Pour autant, et ce sera l'occasion de revenir sur l'articulation entre les textes " répressifs " et la loi de 1964, ce qui fournira une conclusion, il convient de replacer les redevances dues au titre de la pollution dans leur cadre exact.

Dans la réalité, le rejet de substances polluantes est interdit et peut être sanctionné en vertu des dispositions du Code Rural et de la législation relative aux établissements " classés ", parce que tout rejet de ce genre est susceptible de nuire au milieu extérieur.

Pour diverses raisons, ces dispositions n'ont été appliquées que partiellement ou fragmentairement.

La loi de 1964 vise, elle, entre autres objectifs, à éliminer la pollution et à faire en sorte que l'homme dans toutes ses activités ne se trouve pas dans la situation, probable si aucune mesure n'avait 
été décidée, où l'eau fera défaut, aussi bien quantitativement que qualitativement.

Par conséquent, on aurait tort de prétendre que les redevances pollution représentent une charge financière nouvelle, d'autant que dans la plupart des cas, pour ne pas dire dans tous les cas, le montant de ces redevances est inférieur au coût de l'épuration.

L'épuration, c'est-à-dire Ie traitement des effluents des villes, des industries, conçu comme devant supprimer leur pouvoir polluant, est et demeure obligatoire. La loi de 1964 doit permettre une prise de conscience de sa nécessité.

Les Comités de bassin ont refusé le rôle répressif s'ajoutant à celui de l'Administration compétente ou des Tribunaux, que certains voulaient leur voir remplir. Cette attitude est logique, car les motivations des deux actions sont différentes.

En fait, on peut dire que des établissements industriels peuvent être classés en deux catégories : ceux dont les effluents sont traités, qui par conséquent respectent les règlements et qui pour cela supportent une charge financière souvent lourde en voyant en définitive la redevance théorique presque annulée ; et ceux qui ayant échappé aux sanctions prévues, sont astreints à acquitter une redevance pollution.

Remarquons que sauf aspect proprement psychologique, le plafond de la redevance peut être estimé égal au coût de l'épuration : cette remarque a déjà été faite et il n'est pas inconcevable que le montant des redevances ne soit pas dans les années à venir sensiblement majoré par rapport à celui que nous connaissons actuellement.

Enfin, il convient de noter que le problème n'est pas seulement propre à la France : il se présente dans tous les pays industrialisés, avec des formes diverses certes, avec la même acuité cependant.

La politique de l'eau, de l'économie de l'eau est réellement une nécessité. Que son application soulève de grandes difficultés, voire des contradictions, c'est certain. Mais c'est précisément le rôle des organisations professionnelles, des associations spécialisées, au niveau national, au niveau des Comités de bassin, que d'éliminer ces contradictions et de supprimer ces difficultés.

Les intérêts de l'industrie, analysés objectivement, ne permettent pas de remettre en question cette politique de l'eau, sans laquelle l'homme et son industrie sont, à terme, condamnés. 


\section{S u m m a ry}

The purification of waste waters is realized by two methods : trickling by effluent sprinkling and biological treatments.

As far as the latter is concerned, the problem of sludge is solved by incineration.

The law of december 16th 1964 contributes to avoid the pollution of waters wich are necessary in ever increasing quantities.

Water politics have become a real necessity. 